\title{
Zombies Slap Back: Why the Anti-Zombie Parody Does Not Work
}

\begin{abstract}
Duško Prelević
University of Belgrade

DOI: $10.2478 /$ disp-2015-0002

BIBLID [0873-626X (2015) 40; pp. 25-43]

Abstract

In his "anti-zombie argument", Keith Frankish turns the tables on "zombists", forcing them to find an independent argument against the conceivability of anti-zombies. I argue that zombists can shoulder the burden, for there is an important asymmetry between the conceivability of zombies and the conceivability of anti-zombies, which is reflected in the embedding of a totality-clause under the conceivability operator. This makes the anti-zombie argument susceptible to what I call the 'Modified Incompleteness', according to which we cannot conceive of scenarios. In this paper I also argue that conceiving of the zombiesituation is a good starting point for rendering the zombie argument plausible.
\end{abstract}

\section{Keywords}

The zombie argument, the anti-zombie argument, conceivability, scenario, situation.

David Chalmers' zombie argument is one of the most important anti-physicalist arguments in contemporary philosophy of mind, and there is a long-standing debate whether the argument is valid and sound. In this paper, I assess Keith Frankish's "anti-zombie argument" (Frankish 2007), which is intended to be an improved version of the parody of the zombie argument. I argue that Frankish's argument does not prove what he claims it does, for there is an important asymmetry between the conceivability of zombies and the conceivability of anti-zombies. Namely, it will be shown in this paper that zombies might be conceivable by means of conceiving of a zombie situation, while the conceivability of anti-zombies might be formulated only by means of conceiving of an anti-zombie scenario. This is

Disputatio, Vol. VII, No. 40, May 2015

Received: 09/11/2013 Revised: 12/10/2014 Accepted: 24/05/2015 
because the totality-clause, ${ }^{1}$ a second-order sentence that serves to complete a description of a scenario, is contained in the definition of anti-zombies, which is not necessarily so with zombies. If so, then the anti-zombie argument is, in contrast to the zombie argument, liable to what I call the 'Modified Incompleteness', according to which we are not able to conceive of scenarios. Thus, the Modified Incompleteness might serve as an independent argument against the conceivability of anti-zombies and therefore against the anti-zombie argument as well.

After laying out both arguments $(\S 1)$, and showing where the asymmetry lies (\$§2-3), I will suggest a way in which the Modified Incompleteness can be applied against the anti-zombie argument, leaving the zombie argument intact (\$4). Before I start, I want to emphasize that my primary aim in this paper is not to assess one by one all implicit or explicit premises of the zombie argument. Rather, my aim is to show how the zombie argument might be valid and sound (without being redundant), while at the same time the anti-zombie parody might fail. At best, some reasons will be given for favoring the reading of the first premise of the zombie argument that I will assume in the paper and call the 'Non-Idealized Conceivability'.

\section{The Zombie Argument and the Anti-Zombie Parody}

Chalmers offers an elaborated version of the zombie argument. ${ }^{2}$ For the sake of brevity, I will present the details of his argument only to an extent necessary to understand the key notions required for my response to the anti-zombie parody. Chalmers' zombie argument can be formulated in the following way:

(1) Zombies are ideally positively primarily conceivable.

(2) If zombies are ideally positively primarily conceivable, then zombies are metaphysically possible.

\footnotetext{
${ }^{1}$ In the literature, the totality-clause is sometimes called 'that's all clause' or 'stop clause', etc.

${ }^{2}$ See Chalmers (1996: 123; 1999: 473-96; 2002: 198; 2010: 161).
} 
(3) If zombies are metaphysically possible, then consciousness is non-physical.

(4) So consciousness is non-physical.

Here, "zombies" are our physical (relational) duplicates who, unlike us, lack phenomenal consciousness (subjective aspects of conscious experience). A state of affairs is ideally positively primarily conceivable if one can conceive of a counter-actual situation, or a counteractual scenario, which verifies a statement she is conceiving of, ${ }^{3}$ and which is undefeatable by better reasoning (Chalmers 2002, §§1-3; the conceiving of a situation is emphasized in Chalmers 1996: 67). In $\$ 2$ this notion will be articulated in a more detailed way.

Now we can move on to Frankish's anti-zombie argument (Frankish 2007), which is a new and interesting attempt of making parody of the zombie argument. This argument can be formulated in the following way:

(5) Anti-zombies are ideally positively primarily conceivable.

(6) If anti-zombies are ideally positively primarily conceivable, then anti-zombies are metaphysically possible.

(7) If anti-zombies are metaphysically possible, then consciousness is physical.

(8) So consciousness is physical.

Here, "anti-zombies" are considered to be our bare physical duplicates with the same mental and phenomenal properties. Frankish holds that "an object $\mathrm{x}$ is a bare physical duplicate of an object $y$ if $x$ is a physical duplicate of $y$ and has no properties of a non-physical kind" (Frankish 2007: 653).

Frankish sets up his argument so that it makes premise (7) plausible in the same way in which premise (3) is (see Frankish 2007: 654). Namely, both zombists and anti-zombists agree that physical-

\footnotetext{
${ }^{3}$ In contrast to positive conceivability, negative conceivability consists in the conceiving of a scenario (or a situation) that is underdetermined by two mutually exclusive statements (see Chalmers' example with vague predicates in Chalmers 2002: 181).
} 
ists presuppose the validity of the metaphysical supervenience thesis, according to which any two worlds that are physically identical are phenomenally identical as well. But if so, then premise (7) seems plausible because anti-zombies are physically identical to us, while premise (3) seems plausible because metaphysical possibility of zombies shows that the metaphysical supervenience thesis (and so physicalism as well) is false.

Frankish's argument also brings an interesting novelty. Namely, in contrast to its predecessor, the so called 'metamodal argument', the anti-zombie argument does not challenge modal monism (see Frankish 2007: 656), a view according to which the space of logical possibilities (scenarios) is co-extensive with the space of metaphysical possibilities (possible worlds). Yet it will be shown in $\S 4$ that this Frankish's improvement might also be a weakness.

Bearing in mind that zombists cannot accept the anti-zombie parody, let us see how they might try to cope with it. The most reasonable strategy to counter the anti-zombie parody is to reject premise (5). And Chalmers does so as well, arguing that anti-zombies (and physicalism) are, at best, prima facie negatively primarily conceivable (Chalmers 2010: 180). If this is true, then the parody cannot work because prima facie conceivability, unlike ideal conceivability, could be defeated by a better reasoning and it could not satisfy premise (6) (Chalmers 2002: 159).

But why should anti-zombists accept this? If we apply Chalmers' own criterion, namely that in the case of ideal conceivability the burden of proof is on someone who claims that a state of affairs that is conceived of is logically impossible (Chalmers 1996: 96), then Chalmers himself should show why anti-zombies are not ideally (positively primarily) conceivable and where the contradiction lies. This means that the burden of proof now lies on Chalmers' shoulders. ${ }^{4}$ In this way, parody neutralizes the zombie argument.

In fact, Chalmers is skeptical about whether anti-zombies (or physicalism) are ideally positively primarily conceivable. He thinks that we lack good reasons to use the conceivability of anti-zombies (or physicalism) as a premise in the anti-zombie argument. He is convinced of this because "many people have noted that it is very hard

${ }^{4}$ Peter Marton has emphasized this point (Marton 1998). 
to imagine that consciousness is a physical process" (Chalmers 2010: 180). In view of this fact, it seems that we have more reasons to accept (1) as a premise, and once we have done it, we can by a priori reasoning deny premise (5).

Yet this strategy does not work, according to Frankish, because "conceivability is all or nothing" (Frankish 2007: 660), that is, it is not a matter of degree but one of kind. Frankish's reply seems plausible if we distinguish conceivability from similar notions, such as imaginability or even intuition, with which conceivability is easy to conflate. That is because conceivability as such is primarily connected to constructing a consistent description of a situation (or a scenario), and it does not depend on, for example, visualization or self-evidence (which are present in imagination and intuition, respectively) that are arguably a matter of degree. If so, then Chalmers' preferring (1) to (5) is pitting one thesis against another (Frankish 2007: 663). Thus, it seems that Frankish's parody might cope with Chalmers' criticism.

If so, then Frankish suggests that zombists should find an independent argument against the conceivability of anti-zombies. Such an argument should not be as strong as to refute physicalism, for in that case it would render the zombie argument redundant. Frankish thinks that it is not clear that such an argument exists. He explores one option, the so called 'transparency thesis', and shows that it does not succeed, because it makes the zombie argument redundant (see Frankish 2007: 662-4). Frankish's conclusion is that the zombie argument is either unsound (if parody works, then we should reject one of the premises of the zombie argument), or redundant.

In effect, Frankish has turned the tables. Now zombists should find an independent argument against the conceivability of anti-zombies that does not make the zombie argument redundant. Can zombists shoulder the burden?

I think they can, but not in the way Chalmers has done it. Nevertheless, I think that Chalmers' conception of ideal positive primary conceivability, which will be exposed in a more detailed way in $\$ 2$, has resources to cope successfully with the anti-zombie parody. So let us see more carefully which interpretations of ideal positive primary conceivability are at stake in order to find out where exactly the asymmetry between premise (1) and premise (5) lies. 


\section{Two Notions of Ideal Positive Primary Conceivability}

As mentioned in $\S 1$, Chalmers holds that ideal positive primary conceivability might be deployed in terms of conceiving of either a counter-actual situation or a counter-actual scenario. This means that he deals with two notions of ideal positive primary conceivability:

Idealized Conceivability: free of any contingent cognitive limitations, a subject $x$ positively conceives a counter-actual scenario.

Non-Idealized Conceivability: a subject $x$ positively conceives a counter-actual situation, and no better reasoner can refute her.

In both cases, $x$ denotes ourselves or an ordinary member of our community.

Contrary to Chalmers, who does not use this distinction in his reply to the anti-zombie parody, I think that such a distinction is crucial for the whole zombists strategy, as it will be shown in $\S \S 3-4$.

It should be emphasized from the very beginning that both the Idealized and the Non-Idealized Conceivability are different from what might be called 'Non-Ideal Conceivability', or from what Chalmers calls prima facie and secunda facie conceivability (see Chalmers 2002: $\S \S 1-2)$. Namely, prima facie conceivability is, according to Chalmers, typically the case in which a non-expert conceives of a scenario (or a situation), while secunda facie conceivability is the case when an expert does so. Yet, both prima facie and secunda facie conceivability are fallible guides to metaphysical (primary) possibility, because they are refutable by a better reasoning. For example, an expert might think, after careful examination, that something is conceivable, yet such a conviction might be refuted by a better reasoner. An example for this is the Nainve set theory that was refuted famously by Russell's paradox (Chalmers 2002: 155). Ideal conceivability is, in contrast to prima facie and secunda facie conceivability, undefeatable by a better reasoning. Another case of non-ideal conceivability is when one conceives of a situation (or a scenario) that misdescribes the statement she is conceiving of. For example, when one believes that the proof of Goldbach's Conjecture is conceivable simply because she can conceive of a mathematician announcing a proof of Goldbach's Conjecture instead of conceiving the proof of Goldbach's Conjecture 
itself (Chalmers 2002: 160). Given that the Idealized Conceivability and the Non-Idealized Conceivability are the two notions of ideal conceivability, they are different from prima facie and secunda facie conceivability.

Now we can turn to the distinction between situations and scenarios. Chalmers holds that a situation is "a configuration of objects and properties" (Chalmers 2002: 150). A situation is only a part of scenario, which is considered to be "a maximally specific epistemic hypothesis" or an epistemically possible world (Chalmers 2006: 81). To conceive of a scenario, one should provide a "complete qualitative description" of the world she is conceiving of (Chalmers 2002: 177). Chalmers says that a complete description of the world requires enumerating particular facts and giving indexical information; to accept that this is indeed a complete description, we must accept a secondorder totality-clause (Chalmers 1996: 41), namely, that the enumerated particular facts are all the facts in the world. This in fact reflects a Russellian idea that enumerating particular facts is not sufficient for reaching an ontological inventory of the world that one aims to provide (Russell 2010). Thus, the totality-clause is introduced for the reason of completeness, because without this second-order clause one would not be able to determine whether her description might be expanded non-trivially by some other particular facts or not, that is, whether such a description might be filled with new details.

In contrast to scenarios, situations are not complete and do not contain the totality-clause. They can always be expanded non-trivially by adding a new detail. Bearing this in mind, the crucial difference between the Idealized and the Non-Idealized Conceivability is that in the former the totality-clause is embedded under the conceivability operator, while in the latter it is not.

From our perspective, the Idealized and the Non-Idealized Conceivability are not extensionally equivalent in cases in which the totality-clause might be but need not be embedded under the conceivability operator. This is because one might be able to conceive of a situation without being able to conceive of a scenario. On the other hand, from the perspective of an omniscient conceiver it might be that the Idealized and the Non-Idealized Conceivability are extensionally equivalent, yet Chalmers does not appeal to the perspective of a omniscient thinker, because he wants to avoid the trivialization 
of conceivability arguments (Chalmers 2002: 148-9). In the cases in which the totality-clause is essentially involved in a description one is conceiving of, ${ }^{5}$ the description might be conceivable only as a scenario and not as a situation: in such a case, the Idealized and the Non-Idealized Conceivability are not extensionally equivalent. If the abovementioned considerations are correct, the premise (1) might be read in two different ways:

(1a) Zombies are Idealizedly conceivable.

(1b) Zombies are Non-Idealizedly conceivable.

Similarly, (5) might be prima facie read in two different ways too:

(5a) Anti-Zombies are Idealizedly conceivable.

(5b) Anti-Zombies are Non-Idealizedly conceivable.

However, it will be argued in the next section that (5b) is not available for zombists.

\section{On the Asymmetry between Conceiving of Zombies and Conceiving of Anti-Zombies}

Let us turn to the question about whether zombies and anti-zombies are conceivable in the same way. Here, Frankish gives us a hint. Namely, he notices an asymmetry between the conceiving of zombies and the conceiving of anti-zombies, yet he does not see the difference as an insurmountable obstacle to his argument. He says:

It might be objected that there is an asymmetry between (1) and (5), ${ }^{6}$ in that the latter requires us to embed a totality-clause ("no further properties of non-physical kind') under the conceivability operator, whereas the former does not. However, it is not clear that this make (5) less plausible, and both premises are on a par to the extent that they both require us to conceive the absence of something - phenomenal properties in one case, non-physical properties in the other. (Frankish 2007: 654)

${ }^{5}$ It will be argued in this paper that the description of anti-zombies belongs to this category.

${ }^{6}$ In this paper, these are premises (1) and (5) too. 
I think this is a red herring. Of course, for finding an asymmetry between (1) and (5) it is important to see whether conceiving of the absence of something is a case of negative conceivability ${ }^{7}$ or not. For example, if zombies are positively conceivable, while anti-zombies are only negatively conceivable, we could apply 'the inscrutability of truth' objection against the anti-zombie argument, leaving the zombie argument intact. ${ }^{8}$ This objection would serve as an independent argument against the second premise of the anti-zombie argument. Yet Frankish remarks convincingly that both the conceivability of zombies and the conceivability of anti-zombies rest on conceiving of the absence of something. Thus, the asymmetry between (1) and (5), regarding the scope of the conceivability operator, does not bother him. Nevertheless, he ignores an important detail. He is silent on the question whether embedding the totality-clause under the conceivability operator has any connection with the ideal positive primary conceivability, or more precisely, with its two readings - the Idealized and the Non-Idealized Conceivability (laid out in \$2). Here, I think the asymmetry between the conceivability of zombies and the conceivability of anti-zombies seems obvious.

I argue that the conceivability of zombies might be spelled out both in the Idealized and the Non-Idealized sense, while the conceivability of anti-zombies might be spelled out in the Idealized sense only. That is so because the totality-clause, which is used to describe scenarios only, is contained in the definition of anti-zombies, while zombies do not require the totality-clause. In §4 I will exploit this difference.

In order to see why the conceivability of anti-zombies might be understood in the Idealized sense only, let us suppose (for the sake of argument) that we can conceive of the anti-zombie situation. Can it be expanded in a non-trivial way? It seems that it cannot, because

\footnotetext{
${ }^{7}$ The thesis, according to which conceiving of the absence of conscious experience is a case of negative conceivability, appears in Ashwell (2002: 87-93) and Marcus 2004; the opposite view is found in Alter 2007 and Chalmers (2010: 157).

${ }^{8}$ Inscrutability of truth is the thesis that a complete description of a world does not entail all truths about the world. See Chalmers (2002: §8) on the relation between inscrutability and negative conceivability.
} 
it has already been determined that whatever is referred to in the description of the anti-zombie situation has a physical nature, that is, we have already constructed "a complete qualitative description" of the world (see $\$ 2$ ). In other words, by expanding the anti-zombie situation (for example, by introducing ghosts, non-physical ectoplasm, etc.) we would obviously get a world that is not the antizombie world, because not everything in that world would be of a physical kind.

As for zombies, they might be deployed both in terms of Idealized and Non-Idealized Conceivability, because the zombie situation can be expanded non-trivially in many ways. For example, we can expand it by adding a fact that there is no non-physical ectoplasm, a fact that there are no ghosts, angels, and other negative facts. The nature of non-physical ectoplasm, ghosts and angels is not determined beforehand as being of a physical kind, as is in the case with antizombies. Rather, these entities are considered to be of a non-physical kind. So it seems that the zombie situation, according to which the physical is not a sufficient condition for phenomenal consciousness, could be embedded quite coherently into a hypothetical scenario that contains, for example, non-physical ectoplasm or ghosts.

However, although Frankish's actual definitions of anti-zombies require worlds and scenarios (Frankish 2007: 653), someone could still have certain doubts whether anti-zombies are conceivable in the Idealized sense only. After all, one can say that it is sufficient to conceive of a particular anti-zombie situation, that is, the bare physical duplicate of a spatio-temporally finite part of our world. Or, to put the objection in a slightly different way, one can try to go from partial conceivability to the metaphysical possibility of anti-zombies. ${ }^{9}$ If anti-zombies are conceivable both in the Idealized and the NonIdealized sense, then the anti-zombie parody would still work, for no important difference between the conceivability of zombies and the conceivability of anti-zombies would be revealed.

This is a natural move for anti-zombists, yet I think that zombists can defend their view against it successfully. The zombist defense can be grounded on a proper understanding of situations and scenarios in both the zombie and the anti-zombie argument.

${ }^{9}$ Thanks to an anonymous referee for raising this question. 
We have seen in $\S 2$ that Chalmers describes situation as a part of a scenario, and as "a configuration of objects and properties". However, it is unclear in which sense he understands these phrases. For example, according to Yablo, situations can be thought of as spatial, temporal or ontological parts of the world. Possible worlds are complete in every respect, while situations are not (see Yablo 1993: 28). So it seems that situations, in contrast to scenarios, can be complete in one sense and incomplete in another, or can be incomplete in every respect. We have already seen that the crucial distinction between situations and scenarios is that the former could be expanded non-trivially, while the latter cannot.

It seems plausible to consider the zombie situation spatio-temporally complete. Zombies are our physical duplicates that have the same physical (relational) properties that we have, yet they lack phenomenal consciousness. In our debate, 'physical' means something like 'whatever completed physical theory (for example., the theory of everything) says'. Physical theories do not have spatio-temporal constraints, and physical laws should have universal validity. This is usually taken for granted in the debate over physicalism.

Physicalists believe that a physical theory is capable of explaining the whole nature of the world. Namely, spatio-temporal completeness does not ensure ontological completeness, because it is still left open whether the completed physics is capable of explaining everything in the world, or whether there is something 'over and above' the physical. Physicalists say that there is nothing over and above the physical, while their opponents disagree. Here, the issue is the ontological completeness of the physical description of the world, and this is exactly a matter of dispute between zombists and antizombists.

As for the zombie situation, it is sufficient to say that at least one arbitrary phenomenal truth about our universe is not necessitated by the physical base (see, for example, Chalmers 2010: 142), without supposing anything about other phenomenal truths, or about properties of some other kind. Thus, the zombie situation is ontologically incomplete, and we can fill it with new details in various ways, as mentioned above. In order to complete the situation, we need the totality-clause that excludes entities of that sort. In that case, we would get the zombie scenario. Bearing this in mind, zombists can 
insist that the zombie situation is spatio-temporally complete, yet ontologically incomplete.

If the zombie situation should be considered spatio-temporally complete, then the anti-zombie "situation" should be considered spatio-temporally complete too, for anti-zombies are zombies' (as well as our) physical duplicates. But, in contrast to the zombie situation, the anti-zombie "situation" is ontologically complete as well, for it already presupposes that every instantiated property in the world is of a physical kind. Here, we know in advance that everything is physical. So the anti-zombie situation is both spatio-temporally and ontologically complete.

Therefore, the anti-zombie "situation" is not a situation at all, for it is complete in every respect. At least, the burden of proof is on someone who tries to show that we can conceive of the anti-zombie situation; she must show in which sense the anti-zombie situation can be incomplete, that is, how anti-zombies are Non-Idealizedly conceivable.

This point can be expressed more clearly if we recall that Frankish himself presupposes the metaphysical supervenience for the antizombie argument to work (see $§ 1)$. However, according to the standard meaning of the supervenience relation, the fundamental level is such that nothing outside it could exist in some other way except by supervening on the fundamental. Therefore, the very concept of supervenience requires the totality-clause: We cannot even define an anti-zombie in the anti-zombie parody without the totality-clause, because otherwise we cannot get the difference between the relevant cases in which the non-fundamental level (phenomenal consciousness in our case) supervenes on the physical, and the situation in which it is just added as something independent. This turns us back to the claim that the totality-clause is essentially involved in Frankish's parody, and that one can spell out the conceivability of anti-zombies only in terms of the Idealized Conceivability, and not in terms of the Non-Idealized Conceivability. In other words, Frankish's purported description of anti-zombies requires the totality-clause, which suggests that he needs scenarios in his argument instead of situations.

There is another worry on whether the anti-zombie situation is really complete or not. Namely, it seems possible, at least prima facie, to expand the anti-zombie situation by many sentences that describe 
particular facts, such as 'The author of this manuscript had a dinner on May $14^{\text {th }}$ at 7 p.m.,${ }^{10}$ etc. Here, the worry is on whether the totality-clause that Frankish uses in his argument is the same totality-clause that Chalmers uses in constructing scenarios and possible worlds. If these two totality-clauses are not equivalent, then it seems that there is a sense in which anti-zombies are, like zombies, NonIdealizedly conceivable, which would save the symmetry between premises (1) and (5).

Yet it seems that this objection might be avoided if we look more carefully what Frankish's totality-clause means. Namely, it seems plausible to say that Frankish's totality-clause 'All further properties are of a physical kind' refers to all existing, or, more precisely, all instantiated properties of a counter-actual anti-zombie world. Bearing in mind that those properties create (together with objects) particular facts, Frankish's totality-clause, like Chalmers', encompasses all particular facts of the anti-zombie scenario. Simply put, the totalityclause is a second-order sentence about first-order sentences that describe a world, so it can be applied only to the facts about the world that are fixed. In that respect, one and the same totality-clause is present both in the zombie argument and the anti-zombie parody. If so, the conceivability of anti-zombies can be understood in the sense of Idealized Conceivability only.

If these considerations are correct, that is, if the conceivability of zombies might be deployed both in terms of the Idealized and the Non-Idealized sense of ideal positive primary conceivability, while the conceivability of anti-zombies might be deployed in the Idealized sense only, then an independent argument against the conceivability of anti-zombies is on the horizon: It is possible to reject the Idealized Conceivability and to keep the Non-Idealized Conceivability. ${ }^{11}$ But a simplistic denial is not enough. We need an independent argument

${ }^{10}$ Thanks to an anonymous referee for drawing my attention to this problem.

${ }^{11}$ Bearing in mind that ' $p$ is conceivable' is usually understood as 'non- $p$ is not a priori', it is not necessary to deny the Idealized Conceivability in all cases. For example, one might still claim that the Idealized Conceivability is applicable to logical and mathematical truths, which are knowable a priori. The conceivability of zombies, as well as the conceivability of anti-zombies, does not belong to this category. 
against the Idealized and for the Non-Idealized Conceivability. In the next section I develop such an argument.

\section{Modified Incompleteness}

There is an objection against our pretensions to ascribe ourselves omniscient abilities, as well as against any epistemology that posits such exaggerated claims. The objection is simple: We are not in the position of God and our cognitive capacities are limited. Therefore, every good epistemology should incorporate our real-life cognitive capacities, not idealized ones. ${ }^{12}$ One way to put this point is by using what Rebecca Hanrahan calls the 'incompleteness objection' (Incompleteness) (Hanrahan 2009: 389), which contains two relevant ideas. The first idea is that we are not able to conceive of scenarios, because it would require constructing a complete and coherent set of propositions that is infinitely large, and in which a proposition that we are conceiving of is embedded coherently. It seems that conceiving of such a scenario is beyond our capacities. This claim is directed against the Idealized Conceivability, and if true, then both the anti-zombie parody and the Idealized Conceivability reading of the zombie argument fail, leaving hope for the Non-Idealized Conceivability reading of the zombie argument. Yet the second idea of the Incompleteness is directed against the Non-Idealized Conceivability reading. Namely, it seems that the conceiving of a situation does not guarantee that by expanding our system of beliefs we will not get a contradiction. Therefore, it is inappropriate to accept the Incompleteness in its entirety, ${ }^{13}$ because it would undermine the zombie argument as well. ${ }^{14}$

${ }^{12}$ See, e.g., BonJour (1980: 66) and Hanrahan (2009: 390), among others.

${ }^{13}$ In what follows, it will be shown that the Incompleteness does not seem plausible as well, because the Non-Idealized Conceivability is a good starting point for rendering the conceivability arguments plausible.

${ }^{14}$ A variation of the Incompleteness is the so-called Standard Objection, which might be understood as a dilemma in which the first horn is that the NonIdealized Conceivability is not a reliable guide to metaphysical possibility, while the second horn is that the Idealized Conceivability is not accessible to us (see, for example, Worley 2003; Roca-Royes 2011). The Incompleteness is a stronger 
But even if the Incompleteness is not acceptable as a whole, not every part of it should be rejected. We can propose the Modified Incompleteness, which questions the Idealized Conceivability and leaves the Non-Idealized Conceivability untouched. It seems that Chalmers is well aware of this, because he thinks that we can construct successfully the zombie argument by conceiving of a situation, which is likely within our means, without any further requirements concerning our conceivability of a scenario (Chalmers 1996: 67). Thus, the zombie argument can be reconstructed in the following way:

(1') (=1b) Zombies are Non-Ideliazedly conceivable.

(2') If zombies are Non-Idealizedly conceivable, then there is a zombie-scenario.

(3') If there is a zombie scenario, then zombies are metaphysically possible.

$\left(4^{\prime}\right)(=3)$ If zombies are metaphysically possible, then consciousness is non-physical.

(5’) (=4) So the consciousness is not physical.

Then by assuming modal monism, ${ }^{15}$ which was defined in $\S 1$, we can justify premise (3'), and the zombie argument will go through. In fact, it seems plausible that Chalmers uses the distinction between situations and scenarios in order to answer the Incompleteness. ${ }^{16}$ But how might (2') be justified?

Some places in Chalmers' work might suggest that he provides an argument for premise (2'). For example, we have seen in $\S 1$ that Chalmers understands ideal conceivability as undefeatability by a better reasoning, which suggests that ideal conceivability is a rational notion (Chalmers 2002: §1). In view of this fact, the Non-Idealized Conceivability entitles us to think that there is a scenario such that a situation that we are conceiving of might be coherently embedded

claim: it is a conjunction of the two horns of the Standard Objection.

${ }^{15}$ It was noticed in $\S 1$ that both sides in our debate assume modal monism.

${ }^{16}$ Or the Standard Objection; see footnote 13. 
into. Thus, accepting premise (2') seems to be a rational solution. Namely, Chalmers' ideal conceivability is defined in such a way that the burden of proof is on a skeptic, who should find an underlying contradiction in a situation that one is conceiving of. In the case of zombies, the burden of proof is on those who claim that the zombie situation cannot be completed up to a scenario in a coherent way, and such a burden is hard to shoulder.

It is also worth noticing that the existence of a zombie scenario does not commit us to conceive of such a scenario, because such a possibility is left to an omniscient thinker, if he exists. Sometimes, Chalmers appeals to (hypothetical) God's omnipotence in order to illustrate that conceivability should be in accordance with logicoconceptual possibility (Chalmers 1996: 35). In that respect, logical possibility is a boundary of God's omnipotence. Therefore, when we conceive of the zombie situation in the sense of the Non-Idealized Conceivability, we have a good reason to think that God could have ensured (hypothetically) ${ }^{17}$ that the situation we conceived of was embedded coherently in a corresponding zombie scenario, had he so chosen. Given that both zombists and anti-zombists have already accepted modal monism as a premise (see §1), they should also accept that God (hypothetically) can do only what is logically and metaphysically possible. So, appealing to God's omnipotence can serve as an illustration that supports premise (2').

These considerations suggest that the zombie argument can be defended without appealing to the Idealized Conceivability, because the Non-Idealized Conceivability is a sort of ideal conceivability that is relevant in justifying conceivability arguments.

We have seen from the considerations above how modal monism, which both zombists and anti-zombists accept in the current debate, enables zombists to avoid the Incompleteness. Yet the objection against the Idealized Conceivability is still in play, because we have

${ }^{17}$ This means that the validity of conceivability arguments does not depend on the existence of an ideal conceiver. Namely, Chalmers' primary conceivability can be defined by means of apriority and logico-conceptual possibility, which are probably mind-independent and mind-accessible at the same time. Here, mathematics can serve as an illustration, because it seems plausible that mathematical truths are a priori, yet it does not follow from this that we are capable of proving them all. 
adopted the Modified Incompleteness instead of the Incompleteness. Here, only the zombie argument could benefit, for it, in contrast to the anti-zombie parody, can be read in the Non-Idealized sense. Thus, if the Modified Incompleteness is true, the anti-zombie argument does not work, while there is still a hope for the zombie argument to succeed. Because of this, the anti-zombie argument is not a successful instance of the conceivability argument, and therefore it should be rejected.

Finally, we should briefly raise the question whether the Modified Incompleteness makes the zombie argument redundant. We can easily see that this is not the case, for the Modified Incompleteness questions only the conceivability of anti-zombies and not physicalism as such. Namely, (weak) modal rationalists claim that (ideal positive primary) conceivability entails possibility. They are not obliged to claim that inconceivability (that is, in the sense of not being ideally positively primarily conceivable) entails impossibility, for these two theses are independent of each other (see, for example, Casullo 1979: 212). Modal rationalists can grant that something could be inconceivable due to our cognitive limitations and nevertheless possible. But this has no relevance for the reliability of our modal beliefs. It is still possible that everything we properly conceive of is ipso facto possible. Bearing this in mind, the Modified Incompleteness goes counter to the conceivability of anti-zombies, but not to modal rationalism or physicalism. Physicalists (for example, type-B physicalists in Chalmers' terminology) could still claim that physicalism is inconceivable (or not ideally positively primarily conceivable), yet metaphysically possible, and therefore true. Namely, physicalism is usually formalized as $\square(P T I \rightarrow Q)$, where $P$ is a physical description (physical facts, including the laws of nature) of the world, $T$ is the totality-clause, $I$ is indexical information, and $Q$ is a phenomenal truth (see Chalmers 2010: 142, 161). Now, if physicalism is possible, that is, if $\diamond \square(P T I \rightarrow Q)$ is true, then, by the theorem of S5 system $\diamond \square p \rightarrow \square p$ and modus ponens, we can infer that physicalism is true (that is, that $\square(P T I \rightarrow Q)$ is true; cf. Frankish 2007: 656).

But then the zombie argument, if valid and sound, could finally refute physicalism, for it shows that physicalism is false and therefore (by modus tollens) metaphysically impossible. Thus, the Modified Incompleteness does not make the zombie argument redundant. 


\section{Conclusion}

If previous considerations are correct, the anti-zombie parody does not succeed. The refined the zombie argument, based on the Modified Incompleteness, may cope with its anti-zombie antipode. Yet the anti-zombie parody reveals an important point about the nature of conceivability. It shows that zombists should be satisfied with the Non-Idealized Conceivability only, which is a quite acceptable solution, at least in the context of the present discussion in which modal monism has been presupposed. Thus, we can consider the anti-zombie parody as a useful heuristic device: It pushes us to use the NonIdealized Conceivability in order to make conceivability arguments plausible. $^{18}$

Duško Prelević Faculty of Philosophy University of Belgrade Čika Ljubina 18-20, 11000 Belgrade, Serbia dusko.prelevic@f.bg.ac.rs

\section{References}

Alter, Torin. 2007. Imagining Subjective Absence: Marcus on Zombies. Disputatio 22: $91-100$

Ashwell, Lauren. 2002. Conceivability and Modal Error. MA Thesis. University of Auckland.

BonJour, Laurence. 1980. Externalist Theories of Knowledge. Midwest Studies in Philosophy 5: 53-73.

Casullo, Albert. 1979. Reid and Mill on Hume's Maxim of Conceivability. Analysis 39: 212-219.

Chalmers, David. 1996. The Conscious Mind: In Search of a Fundamental Theory. Oxford: Oxford University Press.

Chalmers, David. 1999. Materialism and Metaphysics of Modality. Philosophy and Phenomenological Research 59: 473-496.

Chalmers, David. 2002. Does Conceivability Entail Possibility? In Conceivability and Possibility, ed. by Tamar Gendler and John Hawthorne. Oxford: Oxford University Press.

Chalmers, David. 2010. The Two-Dimensional Argument against Materialism.

${ }^{18}$ I would like to thank Miloš Arsenijević and Andrej Jandrić for stimulating discussions, and Sonia Roca-Royes, Daniel Kostić, Slobodan Perović, and three anonymous referees for valuable comments on earlier drafts of this paper. This research was supported by Ministry of Education, Science and Technological Development of the Republic of Serbia (project: Logico-epistemological foundations of science and metaphysics, No. 179067). 
In The Character of Consciousness. Oxford: Oxford University Press.

Frankish, Keith. 2007. The Anti-Zombie Argument. The Philosophical Quarterly 57: 650-666.

Hanrahan, Rebecca. 2009. Getting God Out of (Our) Modal Business. SOPHIA 48: 379-91.

Marcus, Eric. 2004. Why Zombies are Inconceivable. Australasian Journal of Philosophy 82: 477-490.

Marton, Peter. 1998. Zombies versus Materialists: The Battle for Conceivability, Southwest Philosophy Review 14: 131-138.

Roca-Royes, Sonia. 2011. Conceivability and De Re Modal Knowledge. Nous 45: 22-49.

Russell, Bertrand. 2010. The Philosophy of Logical Atomism. London and New York: Routledge.

Worley, Sara. 2003. Conceivability, Possibility and Physicalism. Analysis 63: 15-23.

Yablo, Stephen. 1993. Is Conceivability a Guide to Possibility? Philosophy and Phenomenological Research 53: 1-42. 